Copyright by the Optical Society of America. Krishna Ramadurai, Christopher L. Cromer, Xiaoyu Li, Roop L. Mahajan, and John H. Lehman, "Foam-based optical absorber for high-power laser radiometry," Appl. Opt. 46, 8268-8271 (2007); doi: 10.1364/ao.46.008268

\title{
Foam-based optical absorber for high-power laser radiometry
}

\author{
Krishna Ramadurai, ${ }^{1, \star}$ Christopher L. Cromer, ${ }^{2}$ Xiaoyu Li, ${ }^{2}$ Roop L. Mahajan, ${ }^{3}$ and John H. Lehman ${ }^{2}$ \\ ${ }^{1}$ Department of Mechanical Engineering, 427 UCB, University of Colorado, 1111 Engineering Drive, Boulder, \\ Colorado 80309, USA \\ 2Division of Optoelectronics, National Institute of Standards and Technology (NIST), 325 Broadway, Boulder, \\ Colorado 80305, USA \\ ${ }^{3}$ Department of Engineering Science and Mechanics, Institute for Critical Technologies and Applied Science, \\ and Department of Mechanical Engineering, Virginia Tech, Blacksburg, Virginia 24060, USA
}

${ }^{\star}$ Corresponding author: krishna.ramadurai@gmail.com

Received 10 September 2007; revised 23 October 2007; accepted 25 October 2007; posted 29 October 2007 (Doc. ID 87300); published 26 November 2007

\begin{abstract}
We report damage threshold measurements of novel absorbers comprised of either liquid-cooled silicon carbide or vitreous carbon foams. The measurements demonstrate damage thresholds up to 1.6 $\times 10^{4} \mathrm{~W} / \mathrm{cm}^{2}$ at an incident circular spot size of $2 \mathrm{~mm}$ with an absorbance of $96 \%$ at $1.064 \mu \mathrm{m}$. We present a summary of the damage threshold as a function of the water flow velocity and the absorbance measurements. We also present a qualitative description of a damage mechanism based on a two-phase heat transfer between the foam and the flowing water. (C) 2007 Optical Society of America
\end{abstract}

OCIS codes: $120.0120,120.5630,140.0140$.

\section{Introduction}

High-power lasers are commonly used in optical lithography, laser welding and cutting, laser surgery, and military devices. In all of these applications, an accurate calibration of the incident irradiation is necessary for consistent process control. The next generation of thermal detectors for high-power industrial and military lasers requires operation at incident power densities exceeding $10^{4} \mathrm{~W} / \mathrm{cm}^{2}$, which poses significant measurement challenges.

Most contemporary primary standards for optical radiation providing traceability to SI units are based on the conversion of optical radiation to heat. A laser calorimeter is one type of device that provides this function. The thermal energy or power is measured with a thermal circuit that is calibrated with an electrical heater. National Institute of Standards and Technology (NIST) has relied on laser calorimeters for many years for measurements over a wide range of wavelengths and power levels [1]. An excellent

0003-6935/07/348268-04\$15.00/0

(C) 2007 Optical Society of America review of the various types of calorimeters used is presented by Gunn [2]. Water-cooled calorimeters and power meters are arguably more suited to highpower laser measurements. Such devices measure the temperature rise of the liquid drawing away the heat delivered by the incident radiation, which is correlated to the absorbed power. Smith et al. [3] built a water-cooled high-power laser calorimeter capable of measurements up to 1 MJ. Emmony and Bunn [4] proposed a flowing water calorimeter capable of measurements up to $50 \mathrm{~W}$. Fisk and Gusinow [5] designed a circulated liquid, volume absorbing calorimeter for use over a wide range of wavelengths. Seguin et al. [6] developed a spinning water film power meter for high-power, continuous wave lasers. Although significant advances have been made in high-power laser measurements, to meet the needs of next generation high-power laser devices, we still require improved precision and accuracy from detectors having high absorption efficiency, low thermal mass, high diffusivity, and a low thermal impedance path to the coolant.

The advantage of the direct absorption of laser power with water-cooled foams lies in the high ab- 
sorption efficiency inherent with a porous material, coupled with the ability to dissipate high heat fluxes to the flowing water. The thermal dispersion is enhanced by recirculating eddies that are shed in the wake of the fluid flow through the porous medium. Transport phenomena in porous media have been studied extensively over the years [7], and in the recent past, their applicability to electronic cooling has attracted attention. Novel finned metal foam heat sinks [8-10] have been demonstrated in forced and buoyancy-induced convection. In a separate application, Boomsma et al. [11] considered open-cell metal foam as cooling media for compact heat exchangers and demonstrated significantly lower thermal resistance with aluminum metal foams. Hetsroni et al. [12] observed heat flux dissipation up to 250 $\mathrm{W} / \mathrm{cm}^{2}$ with aluminum metal foams for cooling electron beam transmission windows. These significant advances in the application of foams cooled by forced convection for high-heat flux dissipation support the feasibility of its implementation for high-irradiance lasers. In the present paper, measurements of laser thermal damage on foam samples are presented, along with the mechanism for the onset of damage.

\section{Experimental Details}

The experiments were performed by exposing the foam samples to incrementally increasing laser irradiance. The samples composed of silicon carbide (with porosity $90 \%$ and a pore density of 254 pores per centimeter [100 pores per inch (ppi)]) and vitreous carbon [with porosity $97 \%$ and a pore density of 254 pores per centimeter (100 ppi)] foams, individually housed in a rectangular plastic (polyoxymethylene) block (76 $\mathrm{mm} \times 38 \mathrm{~mm} \times 38 \mathrm{~mm})$, with a rectangular recess $(25 \mathrm{~mm} \times 13 \mathrm{~mm} \times 6.4 \mathrm{~mm})$ for holding the foam. A fused silica window $(75 \mathrm{~mm} \times 40 \mathrm{~mm}$ $\times 6 \mathrm{~mm}$ ) was clamped to the housing above the foam samples to allow focusing of the laser onto the foam sample. Ports on either end of the housing allowed for the introduction of water to cool the sample. The resulting water flow direction was perpendicular to the optical axis, and its mass flow rate was determined by a Coriolis flow meter. Figure 1 shows a cross-section schematic of the foam-based thermal detector. Calibrated commercial thermistors were mounted in the inlet and outlet water channels to measure the change in water temperature upon transit through the sample. The cooling liquid circulated in a closed loop with a heat exchanger to maintain constant inlet temperature, and a speed-controlled positive displacement pump provided constant flow rate. A small amount of fungicide was present in the cooling water to prevent growth of biological material.

The laser damage was measured with a $1.25 \mathrm{~kW}$ $\mathrm{Nd}$ :YAG laser operating at $1.064 \mu \mathrm{m}$. The laser output was focused into a multimode fiber. The fiber output was collimated, directed through an aperture of $10 \mathrm{~mm}$ diameter, and refocused onto the sample. The final focusing lens was positioned to image the

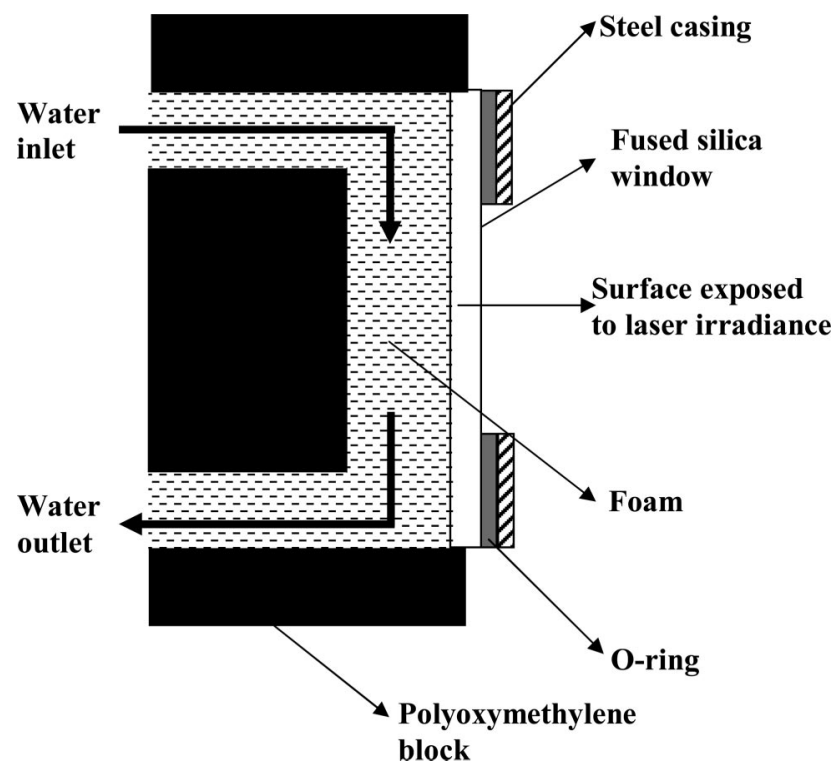

Fig. 1. Two-dimensional top-view cross section of the foam-based thermal detector used in the experiments.

aperture at the sample surface. The beam size at the sample was determined by measuring the aperture diameter and the object as well as the image distances. The spot size in the experiments was determined to be $2 \mathrm{~mm} \pm 0.1 \mathrm{~mm}$. A beam splitter directed a portion of the beam to a power meter to monitor the optical power on the sample. The power at the sample was measured with a NIST-calibrated power meter at a defocused position in the beam. The power absorbed by the sample was calculated as the product of the measured mass flow rate of the water, the change in the water temperature, and the specific heat of the water. The absorption efficiency of the test meter was evaluated as the ratio of the absorbed power to the incident power.

During our experiments, the laser power was incrementally increased until visual damage was observed on the foam, defined by the observation of flashes of light (sparks) on the foam surface. The lowest incident power density at which we observed continuous sparking was referred to as the damage threshold. The damage threshold was measured and documented as a function of increasing water flow rate.

\section{Results and Discussion}

Figure 2 shows the results of the damage threshold measurements for the silicon carbide and vitreous carbon foam as a function of flow rate. The damage threshold for both types of foam materials increased with flow velocity, and the magnitude of the damage threshold in repeated damage experiments varied by $\pm 15 \%$. The threshold increase with increased flow velocity could be due to increased absorption of the incident energy by the flowing water. A maximum threshold of $1.62 \times 10^{4} \mathrm{~W} / \mathrm{cm}^{2}$ was achieved with the silicon carbide foam at a flow velocity of $142 \mathrm{~cm} / \mathrm{s}$, whereas for the vitreous carbon foam, the threshold 


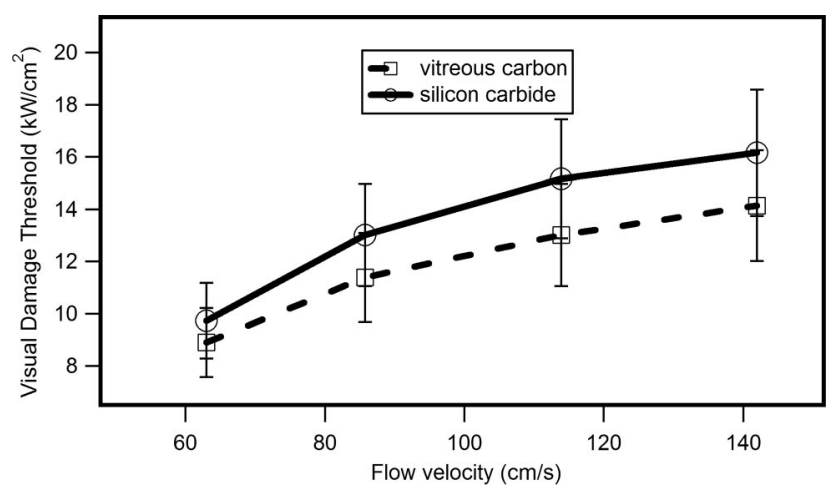

Fig. 2. Visual damage threshold comparison for silicon carbide and vitreous carbon foams as a function of flow velocity.

was $1.41 \times 10^{4} \mathrm{~W} / \mathrm{cm}^{2}$ at the same flow velocity. It was also seen that the silicon carbide foam marginally (within the repeatability limits) outperformed the vitreous carbon foam over the entire range of flow velocities tested.

The absorption efficiency was constant at $96 \%$ over the entire range of flow velocity for each foam material. The fused silica window in front of the foam sample reflects approximately $3 \%$ of the incident radiation. Hence, the effective absorption efficiency for the foam sample could be as high as $99 \%$, which is comparable to that of the best optically absorbing coatings currently available [13]. Of additional significance was that the foam maintained such high values of absorption efficiency even at the damage threshold.

The mechanism of damage to the foam is attributed to phase-change heat transfer. The high pore density and penetration of the beam into the foam resulted in the exposure of a large number of foam fibers in the beam path. The temperature of these fibers rises upon absorption of the laser power, resulting in multiple hot spots. The temperature of the flowing water in contact with these hot spots also increases, and eventually reaches local saturation, resulting in the formation of bubbles that are swept into the water stream. The observation that the surrounding fluid is unsaturated is evident from the temperature measurements at the flow inlet and outlet. Audible boiling noise from the sample reinforced the hypothesis of the growth and the collapse of bubbles. Increasing the input laser power density eventually resulted in the water stream reaching the boiling saturation temperature, which is characterized by persistent bubbles or "bubbly flow." Further increase in the laser power results in the transition from bubbly flow to "slug flow," which is characterized by large vapor pockets [14]. The presence of poorly conducting vapor pockets impedes the heat transfer to the water, thereby resulting in a very large increase in temperature across the pocketed foam fibers. Figures 3(A) and $3(\mathrm{~B})$ show optical images of the unexposed and exposed silicon carbide foam sample. Figure 3(B) depicts damage to the foam as a consequence of high local temperatures.
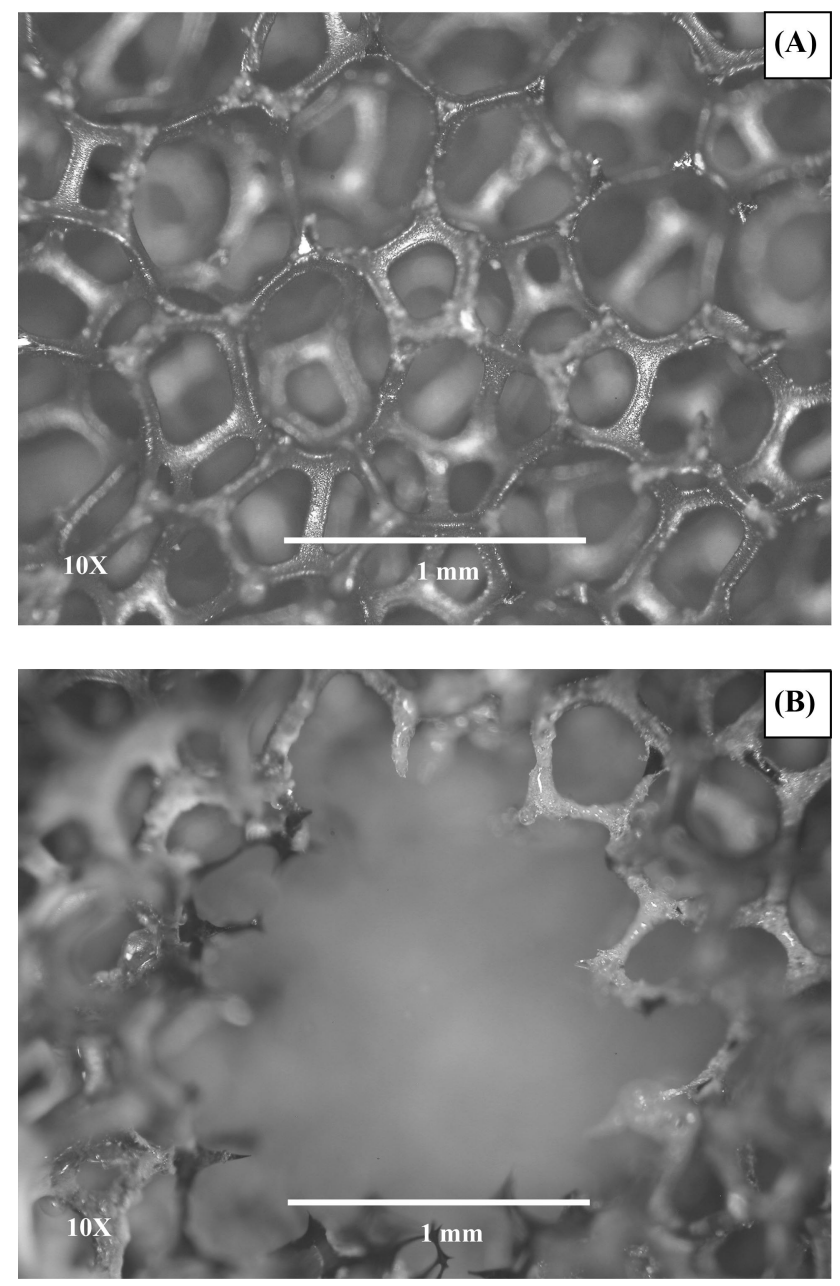

Fig. 3. Optical microscopy image of (A) unexposed and (B) exposed silicon carbide foam sample. The exposed sample shows burnout of the foam sample, as is evident from the gaps in the foam network.

We observed both erosion and removal of fibers from the foam in the irradiated region. The flashing we attribute to damage may be black-body emission from the heated fibers. Both vitreous carbon and silicon carbide oxidize in the presence of water vapor and oxygen, so chemical erosion may also be occurring. The damage threshold of the silicon carbide foam was higher than that of the vitreous carbon foam at the same flow rate. The oxidation temperature of silicon carbide is $\sim 1500 \mathrm{~K}$, whereas that of vitreous carbon is $\sim 1000 \mathrm{~K}$. The difference in the threshold could also be attributed to the relative difference in the material thermal conductivities. The bulk thermal conductivity for the vitreous carbon foam ranged from 0.033 to $0.05 \mathrm{~W} \mathrm{~m}^{-1} \mathrm{~K}^{-1}$, and that of the silicon carbide foam ranges from 1.5 to $2.8 \mathrm{~W} \mathrm{~m}^{-1} \mathrm{~K}^{-1}$. Thus the silicon carbide foam could have a relatively more efficient thermal path for heat flow to the surrounding cooler water stream.

We also observed that the damage threshold was dependent on the laser spot size, with larger diameters resulting in lower damage thresholds. Larger 
beam diameters at the same power density result in larger numbers of hot spots, thereby causing a larger volume of water to reach the saturation temperature. This increase eventually results in filament burnout at relatively lower incident power densities.

\section{Conclusions}

In this work, we evaluated the damage threshold of foams of silicon carbide and vitreous carbon with high incident laser power densities. The damage threshold is as much as $1.62 \times 10^{4} \mathrm{~W} / \mathrm{cm}^{2}$, with absorbance values of over $95 \%$ for a $2 \mathrm{~mm}$ diameter beam on silicon carbide. The damage threshold increased with higher water flow rates and decreased with larger beam size. A mechanism of damage based on the phase-change heat transfer and flow-boiling regimes is proposed. Further studies of the effects of foam density and pore size, and changing the coolant flow direction to parallel to the optical axis, are underway. Thus direct absorption of high-power laser radiation by water-cooled silicon carbide and vitreous carbon foam, by virtue of their high absorbance and high damage threshold, demonstrate great potential for use in high-power laser measurement devices.

\section{References}

1. G. W. Day, "Metrology for the optoelectronics industry," Proc. SPIE 4450, 33-43 (2001).

2. S. R. Gunn, "Calorimetric measurements of laser energy and power,” J. Phys. E 6, 105-114 (1973).
3. R. L. Smith, T. W. Russell, W. E. Case, and A. L. Rasmussen, "A calorimeter for high-power CW lasers," IEEE Trans. Instrum. Meas. IM-21, 434-438 (1972).

4. D. C. Emmony and J. C. S. Bunn, "An absolute calorimeter for the measurement of $\mathrm{CO}_{2}$ laser power," J. Phys. E 9, 621-622 (1976).

5. G. A. Fisk and M. A. Gusinow, "Circulated-liquid calorimeter for the detection of high-power and high-energy pulsed laser signals," Rev. Sci. Instrum. 48, 118-131 (1976).

6. H. J. J. Seguin, V. A. Seguin, A. K. Nath, and J. Radzion, "Spinning water film power meter for high-power cw lasers," Rev. Sci. Instrum. 57, 185-190 (1985).

7. M. Kaviany, Principles of Heat Transfer in Porous Media (Springer-Verlag, 1991).

8. M. L. Hunt and C. L. Tien, "Effect of thermal dispersion on forced-convection in fibrous media," Int. J. Heat Mass Transfer 31, 301-309 (1988).

9. T. J. Lu, H. A. Stone, and M. F. Ashby, "Heat transfer in open-cell metal foams," Acta Mater. 46, 3619-3635 (1998).

10. A. Bhattacharya and R. L. Mahajan, "Finned metal foam heat sinks for electronics cooling in forced convection," J. Electron Packaging 124, 155-163 (2002).

11. K. Boomsma, D. Poulikakos, and F. Zwick, "Metal foams as compact high performance heat exchangers," Mech. Mater. 35, 1161-1176 (2003).

12. G. Hetsroni, M. Gurevich, and R. Rozenblit, "Metal foam heat sink for transmission window," Int. J. Heat Mass Transfer 48, 3793-3803 (2005).

13. W. L. Wolfe and G. J. Zissis, The Infrared Handbook (Environmental Research Institute of Michigan, 1989), pp. 7.787.80 .

14. F. P. Incropera and D. P. Dewitt, Fundamentals of Heat and Mass Transfer, 5th ed. (Wiley, 2002). 